\title{
Implementation algorithm of a generalised plasticity model for swelling clays
}

\author{
M. Sánchez ${ }^{\mathrm{a}, *}$, A. Gens ${ }^{\mathrm{b}}$, L. Guimarães $^{\mathrm{c}}$, S. Olivella ${ }^{\mathrm{b}}$ \\ ${ }^{a}$ Department of Civil Engineering, University of Strathclyde, Glasgow, UK \\ ${ }^{\mathrm{b}}$ Department of Geotechnical Engineering and Geosciences, Universitat Politècnica de Catalunya, (UPC), Barcelona, Spain \\ ${ }^{\mathrm{c}}$ Department of Civil Engineering, Universidade Federal de Pernambuco, Recife, Brazil
}

\section{A R T I C L E I N F O}

\section{Article history:}

Available online 26 September 2008

\section{Keywords:}

Constitutive model

Numerical implementation

Finite elements

Expansive clays

Unsaturated behaviour

Engineered barrier

\begin{abstract}
A B S T R A C T
A multi-mechanism generalisation of Sloan's integration scheme for elasto-plastic laws has been developed in order to implement a double structure model for expansive clays into a FE code. The constitutive model is built on a conceptual approach for unsaturated expansive soils in which the fundamental characteristic is the explicit consideration of the two pore levels often present in expansive clays. The distinction between macro and microstructure provides the opportunity to take into account the dominant phenomena that affect the behaviour of each structural level and the main interactions between them. The model is formulated using concepts of classical and generalised plasticity theories. The integration scheme proposed can deal with the two plastic mechanisms defined in the model and can incorporate the effects of strains, suction and temperature in the stress integration process. A large scale heating test is analysed to check the capabilities of the implemented model to simulate an actual problem involving complex thermo-hydro-mechanical stress paths. The performance of the model has been very satisfactory and the proposed integration scheme has proved to be robust and efficient in solving a highly non-linear coupled problem.
\end{abstract}

(c) 2008 Elsevier Ltd. All rights reserved.

\section{Introduction}

The study of expansive soils has been the subject of increasing interest in the last few years. A major aspect is the possible use of those materials as engineered barriers in radioactive waste repositories. Significant THM (thermo-hydro-mechanical) phenomena take place in the clay barrier and its near field due to the combined actions of heating arising from the canister, and hydration from the surrounding rock. However, the interest in these materials is not limited to nuclear waste disposal applications, but they are present in other engineering problems such as shallow and deep foundations, slopes with stability problems, or desiccation and formation of cracks in soils in arid regions.

Numerical models are useful tools for the design and assessment of geotechnical problems in general and they emerge as indispensable when the interest is focused on performance assessment involving long-term predictions. A typical example is the design of a repository for high level radioactive waste in which it is necessary to predict its THM behaviour over periods lasting thousands of years. Constitutive models able to reproduce correctly clay

\footnotetext{
* Corresponding author. Tel.: +44 141548 3273; fax: +44 1415532066 .

E-mail address: marcelo.sanchez@strath.ac.uk (M. Sánchez).
}

behaviour under the complex THM paths envisaged for the clay barrier are a basic component of that kind of analysis. The clay fabric plays a fundamental role to understand and to reproduce correctly the behaviour of expansive clays as revealed in a number of recent studies [3-7]. Gens and Alonso [3] proposed a general approach to analyze the behaviour of expansive clays in which the fundamental characteristic is the explicit consideration of the two dominant structure levels often present in swelling clays. Elasto-plastic models based on that double structure approach have been widely used as a conceptual framework to describe the behaviour of swelling materials and also to perform analyses at point integration level (i.e. [4-7]). However, their inclusion and exploitation in a finite element program to solve actual boundary value problems has not been done previously.

A refined Euler scheme with automatic sub-stepping and errors control based on Sloan's scheme [1,2] is proposed in this paper in order to implement the double structure model presented in Sánchez et al. [7] in the finite element program CODE_BRIGHT [8]. A feature of the double structure model is that the soil behaviour can be regarded as the result of the joint action of several mechanisms that can act simultaneously. Therefore, the proposed scheme is a multi-mechanism generalisation of Sloan's one [1,2] for the particular conditions of the double structure model. A great advantage of Sloan's scheme is that it has been widely tested in a number of geotechnical problems proving its accuracy, robustness and efficiency. 
The paper is organized as follows: firstly the main components of the model are outlined; secondly, the scheme proposed to implement the constitutive model in the finite element code is described; thirdly, an application case that shows the capabilities of the model is presented; finally, the main conclusions of the work are given.

\section{Model formulation}

Soil fabric plays a crucial role to understand and reproduce the behaviour of expansive clays. The pore size distribution of swelling clays often exhibits two dominant pore sizes. For example Fig. 1 shows the results of mercury intrusion porosimetry tests of statically compacted samples of FEBEX bentonite for two specimens compacted to different values of dry density $\left(\rho_{\mathrm{d}}\right)[6]$. The pore size distribution is clearly bi-modal and the two dominant pores size may be associated with two basic structural levels (Fig. 1): (i) the macrostructure, related to the global arrangements of clay aggregates (i.e. the skeleton of the material), enclosing macropores between them; (ii) the microstructure, which corresponds to the active clay minerals and their vicinity, present inside the aggregates.

In this type of soils there are plastic mechanisms in addition to those observed in non-swelling materials. Those additional mechanisms can be attributed to the interaction between the macrostructure and the microstructure [3]. This kind of irreversible

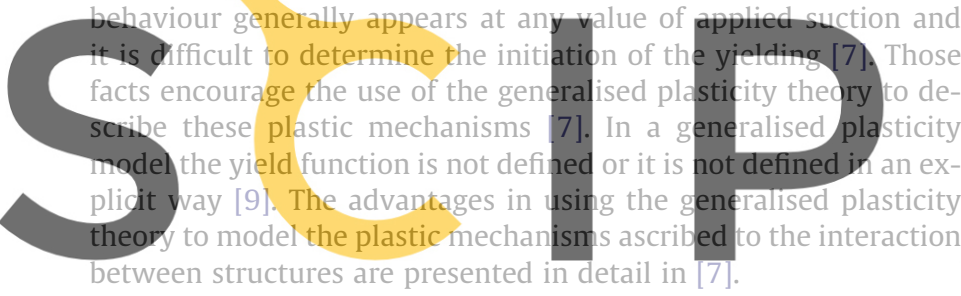

The model is defined in terms of the three stress invariants

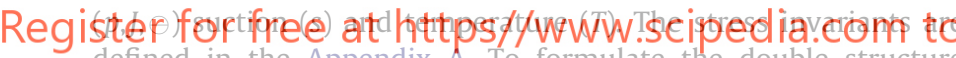
defined in the Appendix A. To formulate the double structure model is necessary to define laws for: (i) the macrostructural level, (ii) the microstructural level and (iii) the interaction between both structural levels. The full mathematical framework is presented in Sanchez et al. [7], herein only the main components of the model are briefly introduced for the sake of completeness. Additional information of the model is presented in the Appendix A. Table 1 presents the notation and nomenclature adopted in this work.

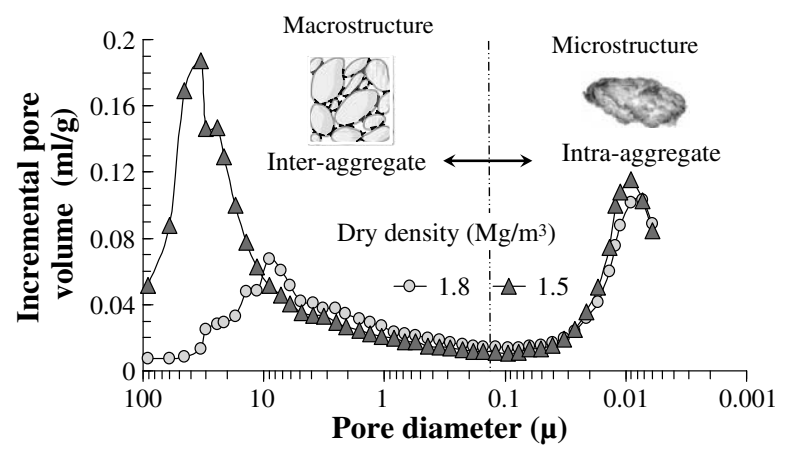

Fig. 1. Distributions of incremental pore volume for two statically compacted samples of FEBEX bentonite [6].

\subsection{Macrostructural model}

The inclusion of this structural level in the analysis allows the consideration of phenomena that affect the skeleton of the material, for instance deformations due to loading and/or collapse of the macrostructure. The BBM (Barcelona Basic Model) has been adopted to describe the macrostructural behaviour [12]. The BBM considers two stress variables to model the unsaturated behaviour: the net stress $(\boldsymbol{\sigma})$ computed as the excess of the total stresses over the gas pressure, and the matric suction $(s)$, computed as the difference between gas pressure and liquid pressure $\left(p_{g}-p_{1}\right)$. Fig. 2a shows the BBM yield surface $\left(F_{L C}\right)$, which is defined as follows:

$F_{L C}=3 J^{2}-\left[\frac{g(\theta)}{g\left(-30^{\circ}\right)}\right]^{2} M^{2}\left(p+p_{s}\right)\left(p_{0}-p\right)=0$

where $M$ is the slope of the critical state, $p_{0}$ is the apparent unsaturated isotropic pre-consolidation pressure at a specific value of suction, and $p_{s}$ considers the dependence of shear strength on suction and temperature. To complete the definition of the yield surface as set out in (1), it is possible, in principle, to adopt any suitable Lode's angle function, $g(\theta)$. For instance, in the CODE_BRIGHT code used in the analysis reported in this paper, the following functions are available: Von Mises (i.e. $g(\theta=$ constant), Mohr-Coulomb and Van Eekelen [13]. Other functions could be considered such as the one suggested in [10], which applies to soils with a friction angle less than $22^{\circ}$ (to ensure a convex yield surface in the deviatoric plane) or the one recommended in [11], which applies to a fric-

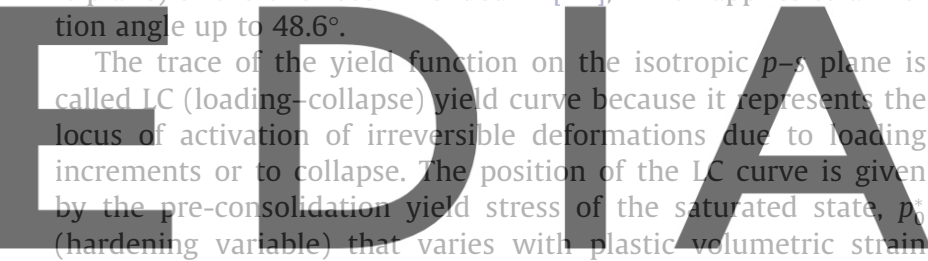

according to the following hardening law

\section{download the $e_{p_{0}}$ version without the watermarck}

where $e$ is the void ratio, $\dot{s}_{v}^{p}$ is the volumetric plastic strain, $x$ is the elastic compression index for changes in $p$ and $\lambda_{(0)}$ is the stiffness parameter for changes in $p$ for virgin states of the soil in saturated condition. In addition, the model is able to describe the reduction of the size of the yield surface and the strength of the material with the increase of temperature, according to the model proposed in Gens [14].

\subsection{Microstructural model}

The microstructure is the seat of the basic physico-chemical phenomena occurring at clay particle level. The strains arising from microstructural phenomena are considered nonlinear elastic and volumetric [3]. The microstructural strains are proportional to the microstructural effective stress $(\hat{p})$ through a microstructural bulk modulus according to

$\hat{p}=p+\chi s \quad(\mathbf{a}) ; \quad \dot{\boldsymbol{\varepsilon}}_{v m}=\frac{\dot{\hat{p}}}{K_{m}}=\frac{\dot{p}}{K_{m}}+\chi \frac{\dot{s}}{K_{m}}$

where the subscript $m$ refers to the microstructural level, the subscript $v$ refers to the volumetric component of the strains, $K_{m}$ is the microstructural bulk modulus and $\chi$ is a parameter normally dependent on the degree of saturation of the microstructure. The parameter $\chi$ is included only to account for the possibility that the microstructure may become unsaturated. However, generally a constant value of $\chi=1$ is adopted, since the micro-structural level, associated with the behaviour of expansive clay particles, is very 
Table 1

Notation

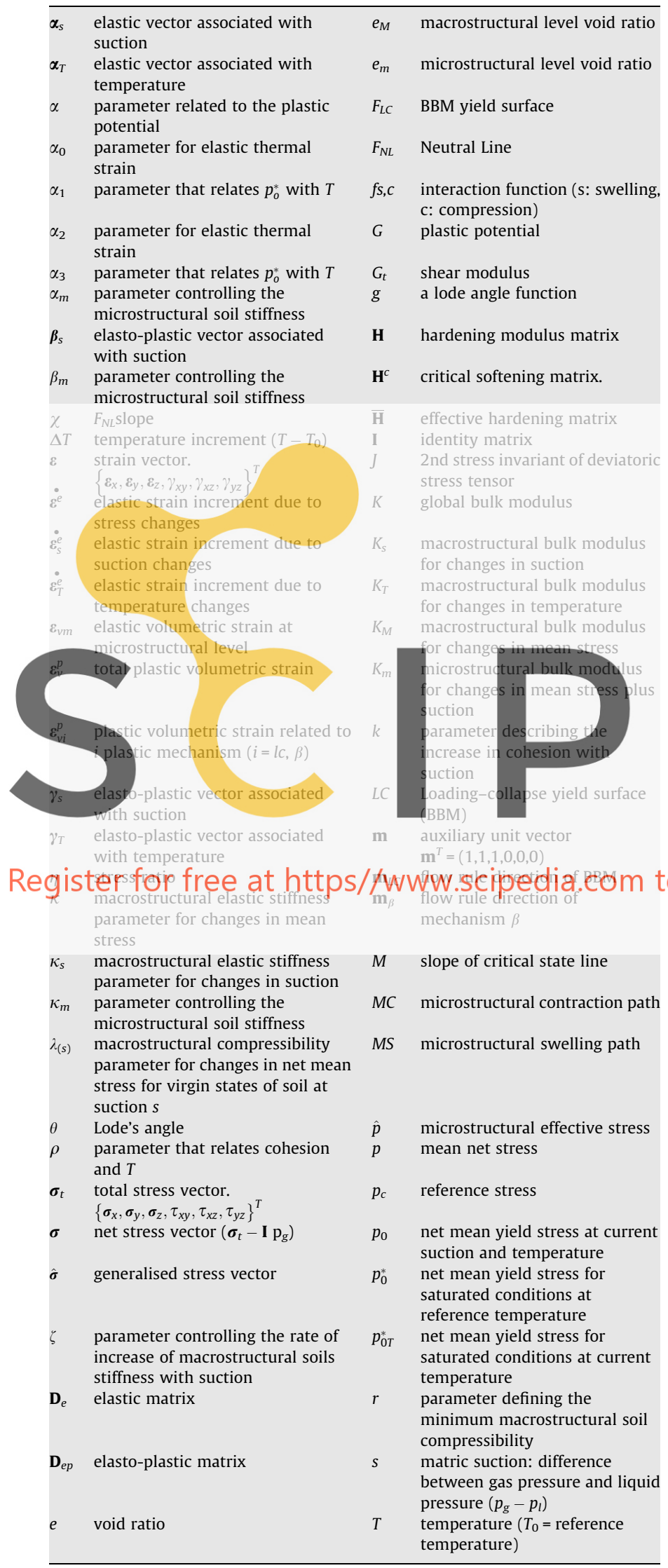

likely to be saturated [3]. In very active expansive clays this assumption can be supported by the high affinity of the active clay minerals by water, which maintain the interlayer space and micropores saturated even at relatively high suction. Under this condition mean effective stress control the mechanical behaviour at microstructural level [3]. Hydraulic equilibrium is assumed between the water potentials of both structural levels, the extension to non equilibrium conditions is presented in $[5,15]$.

The concept of a neutral line (NL) is introduced corresponding to constant $\hat{p}$ and no microstructural deformation (Fig. 2b). The NL divides the $p$-s plane into two parts, defining two main generalized stress paths, which are identified as MC (microstructural contraction) when there is an increase in $\hat{p}$ and MS (microstructural swelling) in the opposite case.

\subsection{Interaction between macro and micro structure}

Based on experimental evidence, it is assumed that the macrostructure is affected by microstructural deformations generally in an irreversible way [3]. A hypothesis of the model is that the plastic deformations of the macrostructure $\left(\boldsymbol{\varepsilon}_{v M}^{p}\right)$ induced by microstructural effects are proportional to the microstructural strains $\left(\boldsymbol{\varepsilon}_{v m}\right)$ according to interaction functions $f$ [7]. The total plastic macrostructural strains (i.e. the sum of the plastic strains induced by the yielding of the macrostructure, $\varepsilon_{L C}^{p}$, plus that induced by the microstructure, $\varepsilon_{v M \rightarrow m}^{p}$ ) are evaluated using (4):

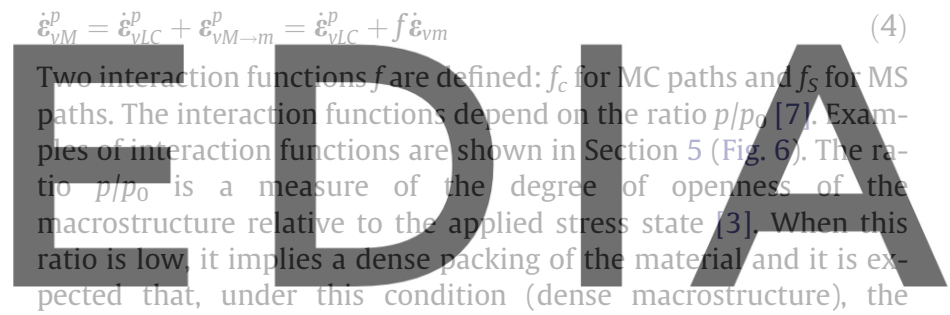
microstructural swelling (MS path) affects strongly the global

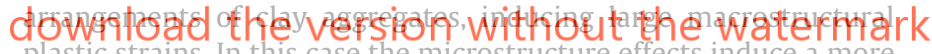
plastic strains. In this case the microstructure effects induce a more open macrostructure, which implies a macrostructural softening. On the other hand, when the microstructure contracts (MC path) the induced macrostructural plastic strains are larger with open macrostructures, that is, for values of $p / p_{0}$ close to 1 . Under this path the clay tends to a more dense state, which implies a hardening of the macrostructure. The result of the coupling between macro and micro levels is reflected in the value of $p_{0}^{*}$, the hardening variable of the macrostructure (Fig. 2a), which depends, through (2), on the total plastic volumetric strain (4). In this way the effect of microstructural processes on the global arrangements of aggregates is taken into account [7].

To describe correctly the soil behaviour, the definition of specific elasto-plastic laws for each domain is required according to the microstructural stress path followed (MC or MS). Generalised plasticity theory can deal with such conditions, allowing the consideration of two directions of different behaviour and the formulation of proper elasto-plastic laws for each region. Thus, a complete description of a generalised model includes the definition of the: (i) loading and unloading direction, (ii) plastic flow direction, and (iii) a plastic modulus [7].

Equivalent to loading/unloading directions in conventional stress/strain formulations, two vectors may be defined at every point of the stress space. One vector indicates the MC direction and the other the MS direction. Given a generalised stress state and stress increment, the criterion to identify the microstructural stress path is specified as (Fig. 2b): 

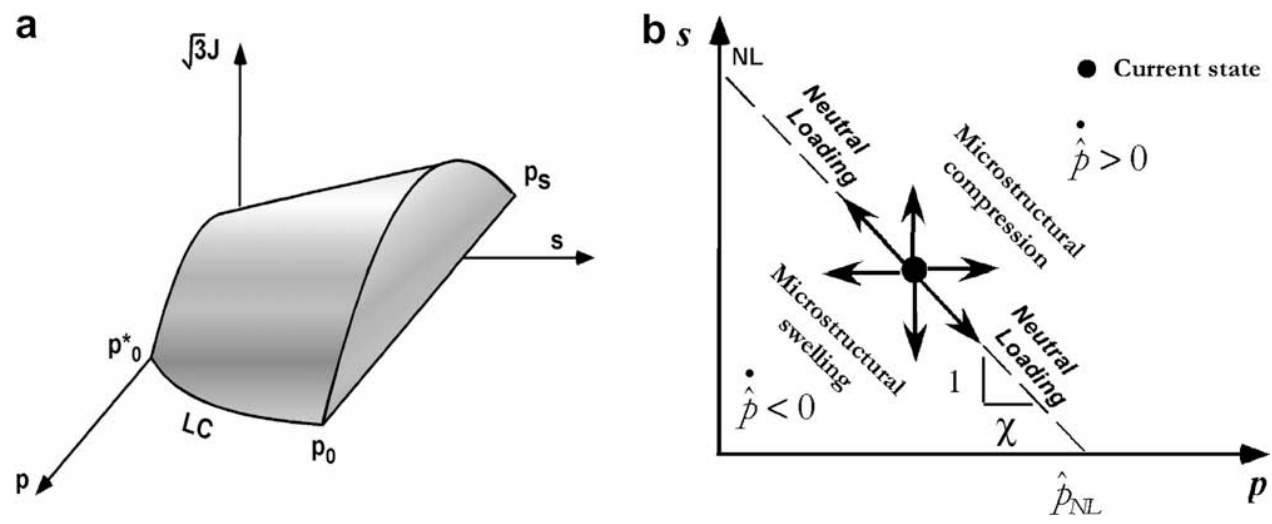

Fig. 2. (a) BBM yield surface. (b) Microstructural load directions on the $p-s$ plane.

\begin{abstract}
Microstructural compression criterion : $\hat{\mathbf{n}}_{C}^{T} \cdot \dot{\hat{\boldsymbol{\sigma}}}^{e}>0(\mathrm{MC}$ path) (5) Microstructural swelling criterion : $\hat{\mathbf{n}}_{S}^{T} \cdot \dot{\hat{\boldsymbol{\sigma}}}^{e}>0$ (MS path) Neutral loading : $\hat{\mathbf{n}}_{\beta}^{T} \cdot \dot{\boldsymbol{\sigma}}^{e}=0$ (NL path)

where $\dot{\hat{\sigma}}^{e}$ is the elastic generalised stress increment, $\hat{\mathbf{n}}_{C}^{T}$ and $\hat{\mathbf{n}}_{s}^{T}$ are the microstructural compression and swelling directions. In [7] the other model ingredients are introduced in detail.
\end{abstract}

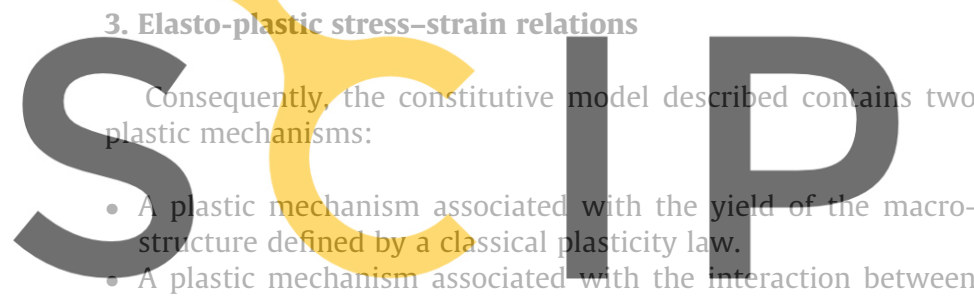
microstructure and macrostructure described by a generalised

\section{Register reficity free at https//www.scipedia.com to}

In classical plasticity theory, it is assumed that the material behaves either as an elastic or an elasto-plastic solid. The yield surface defines the transition from elasticity to plasticity, stress states inside the yield surface are considered as elastic $(F<0)$. In generalised plasticity theory, the state of the material is determined directly from the control variables: generalised stresses, strains and a finite number of internal variables. A process of loading is defined as elastic if the set of internal variables remains unchanged.

In the case of a purely (nonlinear) elastic loading, the stress increment is related to the increment of strains, suction and temperature by the following relationship:

$\dot{\boldsymbol{\sigma}}=\mathbf{D}_{e} \cdot \dot{\boldsymbol{\varepsilon}}^{e}+\alpha_{s} \dot{S}+\alpha_{T} \dot{T}$

where $\mathbf{D}_{e}$ is the global elastic matrix that considers the elastic component of both structural levels. $\boldsymbol{\alpha}_{T}$ and $\boldsymbol{\alpha}_{s}$ are the elastic vectors associated to temperature and suction respectively.

When a loading process is inelastic, the material behaviour is described by elasto-plastic mechanisms that can be activated during the loading process. A multidissipative approach [16] has been adopted to derive the general elasto-plastic relations [7] that can be expressed as follows:

$\dot{\boldsymbol{\sigma}}=\mathbf{D}_{e p} \cdot \dot{\boldsymbol{\varepsilon}}+\gamma_{s} \dot{\boldsymbol{s}}+\gamma_{T} \dot{T}$

where $\boldsymbol{D}_{e p}$ is the global elasto-plastic matrix, $\gamma_{T}$, and $\gamma_{\mathrm{s}}$ are the elasto-plastic vectors associated with temperature and suction, respectively. The expressions for the vectors and matrices in (8) and (9) are presented in the Appendix A.

\section{Model implementation}

A refined explicit Euler method with automatic sub-stepping and errors control has been adopted to implement the model presented in the previous section in the finite element code CODE_BRIGHT. The proposed scheme is a generalisation of algorithm proposed in [1,2], considering the erratum published in [17]. CODE_BRIGHT is a tool designed to analyze numerically coupled THM problems in geological media that allows the performance

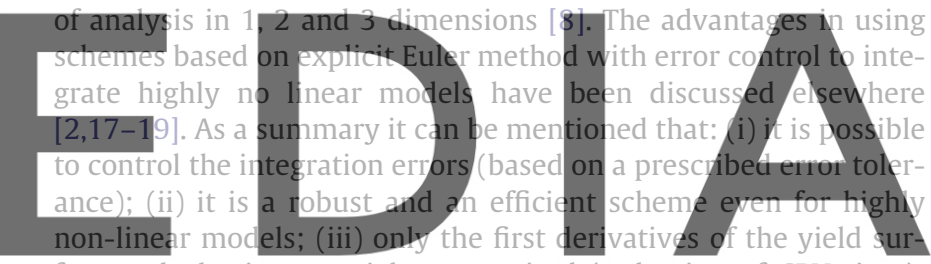

face and plastic potential are required (reduction of CPU time); (iv) this type of scheme provides an open and flexible framework

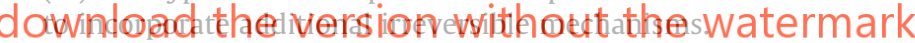

The proposed algorithm can deal with the two plastic mechanisms defined in the model and it can consider the effects of strains, suction and temperature in the stress integration process. Four different cases can be distinguished in relation to the current stress state and the number of irreversible mechanisms active:

- Case I: Behaviour purely (nonlinear) elastic.

- Case II. Only the plastic mechanism associated with the coupling with the two structural levels is active.

- Case III. Only the plastic mechanism associated with the yielding of the macrostructure is active.

- Case IV. The two plastic mechanisms are active simultaneously.

The control variables of the problem are strains, suction and temperature. Following a similar procedure to that proposed in [19], the suction and temperature are treated as additional strain components. For simplicity's sake, the generic name of generalised strains is assigned to this enlarged strain vector (i.e. including strain, suction and temperature). The initial state of the material is given by the initial stresses, the initial values of hardening parameters ( $p_{0}^{*}$ and $\hat{p}_{\mathrm{NL}}$ ) and the initial values of the history variables (i.e. $e_{M}$ and $e_{m}$, macro and micro void ratio respectively). Therefore four internal variables $r$ are considered in these model $\left(p_{0}^{*}, \hat{p}_{\mathrm{NL}}, e_{M}\right.$ and $\left.e_{m}\right)$. Only the main aspects of the implementation scheme are presented in this paper, more details are given in [15].

During a typical iteration of a non-linear problem, the initial state, history variables and the increment of the generalised strains are known at each integration point. The stresses must be numerically updated in order to be consistent with this generalised 
strains increment. The first stage in the procedure is the division of the given generalised strain increment into a number of sub-steps by defining a pseudo time $\tau$ [2]:

$\tau=\frac{\left(t-t_{0}\right)}{\Delta t} \quad 0 \leqslant \tau \leqslant 1$

where $t_{0}$ is the time at the start of the considered increment (global system) and $\left(t_{0}+\Delta t\right)$ is the time at the end of this increment. Below, the main stages to update stresses and history variables are described.

A number of different procedures must be used in the constitutive law integration process. Initially, a generalised strain subincrement inside the LC is assumed (Section 4.1). After the stress integration, it must be verified whether the stress increment still remains inside the main LC yield locus and if this is not the case the yield surface contact point must be determined (Section 4.2). The integration of the strain subincrement going outside the main yield surface must distinguish between the case where only one mechanism is active (Section 4.3) or two mechanism are acting simultaneously (Section 4.4). Finally, the rare case of a purely elastic increment is considered in Section 4.5.

4.1. Integration of the generalised elasto-plastic law (irreversible mechanism associated with the coupling between the two structural levels)

In this section, the case of a stress state that remains inside the LC yield Cu
eralised
ized plas
elasto-pla
no specif
fore, to
is active, curve is examined (Case II). I plasticity model applies. As pexplained. is case, the
alternatined in [
as asumechan
icrostructu
given by [7], the general-
atives to model
med that there is
anism [7]. Therepressed in terms of tolerances, (as required by numerical implementation) as follows:

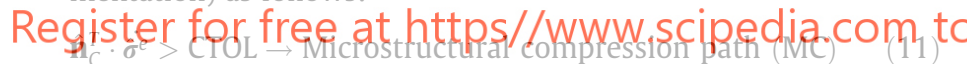

$\hat{\mathbf{n}}_{S}^{T} \cdot \hat{\sigma}^{e}>\mathrm{CTOL} \rightarrow$ Microstructural swelling path $(\mathrm{MS})$

$\left.\hat{\mathbf{n}}_{C}^{T} \cdot \dot{\boldsymbol{\sigma}}^{e}<\mathrm{CTOL}\right\}$

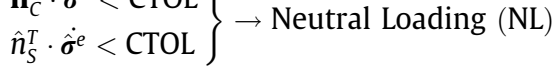

where CTOL is a tolerance associated with the loading criteria of the coupling mechanism between structures.

The Euler's scheme with error control and sub-stepping proposed by Sloan et al. [2] to integrate conventional elasto-plastic laws has been adopted to update the stresses for the generalised plasticity model. In Sloan's algorithm the size of each sub-increment may vary through the integration process, depending on the degree of non-linearity of the constitutive relations. Considering a pseudo time sub-increment in the range $0 \leqslant \Delta \tau_{n} \leqslant 1$, and using the subscript $n-1$ and $n$ to denote quantities computed at the pseudo times $\tau_{n-1}$ and $\tau_{n}=\tau_{n-1}+\Delta \tau_{n}$, the generalised strains for this sub-increment are given by

$\Delta \boldsymbol{\varepsilon}_{n}=\Delta \tau_{n} \Delta \boldsymbol{\varepsilon} \quad(\mathrm{a}) ; \quad \Delta s=\Delta \tau_{n} S \quad(\mathrm{~b}) ; \quad \Delta T=\Delta \tau_{n} T \quad$ (c)

With the explicit Euler method, the stress increments (9) at the end of the pseudo time step $\Delta \tau_{n}$ are evaluated through:

$\Delta \sigma_{1}=\mathbf{D}_{e p_{\left|\sigma_{n-1}, r_{n-1}\right|}} \cdot \Delta \boldsymbol{\varepsilon}_{n}+\gamma_{s\left|\sigma_{n-1}, s_{n-1}, r_{n-1}\right|} \Delta s+\gamma_{T\left|\sigma_{n-1}, T_{n-1}, r_{n-1}\right|} \Delta T$

where $\mathbf{X}_{\left|\sigma_{n-1}, r_{n-1}\right|}$ means that the matrix $\mathbf{X}$ is evaluated at $\boldsymbol{\sigma}_{n-1}$ and $r_{n-1}$. The expressions of $\mathbf{D}_{e p}, \gamma_{s}$ and $\gamma_{T}$ are given in the Appendix A (Eqs. $A(14), A(15)$ and $A(16)$, respectively). The estimated value of stresses and internal variables are obtained from:

$\sigma_{n}=\sigma_{n-1}+\Delta \sigma_{1}$
A more accurate estimation of the stresses increment at the end of the interval $\Delta \tau_{n}$ can be found using the modified Euler procedure:

$\Delta \boldsymbol{\sigma}_{2}=\mathbf{D}_{e p\left|\boldsymbol{\sigma}_{n}, r_{n}\right|} \cdot \Delta \boldsymbol{\varepsilon}_{n}+\gamma_{s\left|\boldsymbol{\sigma}_{n}, s_{n}, r_{n}\right|} \Delta s+\gamma_{T\left|\sigma_{n}, T_{n}, r_{n}\right|} \Delta T$

The following estimation of the stresses and internal variables can then be made using:

$\tilde{\boldsymbol{\sigma}}_{n}=\boldsymbol{\sigma}_{n-1}+\frac{1}{2}\left(\Delta \boldsymbol{\sigma}_{1}+\Delta \boldsymbol{\sigma}_{2}\right)$

The relative error measure $\left(R_{n}\right)$ can be obtained as [1]

$R_{n}=\frac{1}{2} \frac{\left\|\Delta \boldsymbol{\sigma}_{2}-\Delta \boldsymbol{\sigma}_{1}\right\|}{\left\|\tilde{\boldsymbol{\sigma}}_{n}\right\|}$.

The updating of the current sub-increment is accepted if $R_{n}$ is not greater than the prescribed tolerance, STOL, and it is rejected otherwise. If it is rejected, a reduction of the size of the sub-increment is made and the integration process is repeated considering the new (reduced) generalised strain sub-increment (more details are given in Section 4.6). This loop is repeated until the prescribed value of error tolerance is satisfied. When the acceptance condition is met, and before updating the stresses, it is necessary to check whether the new stress state remains inside the LC yield surface as described in Section 4.2. If the stress state still remains in the yield surface, stresses and internal variables are updated according to

$\sigma_{n}=\tilde{\sigma}_{n} \quad(\mathrm{a}) ; \quad r_{n}=\tilde{r}_{n} \quad(\mathrm{~b})$

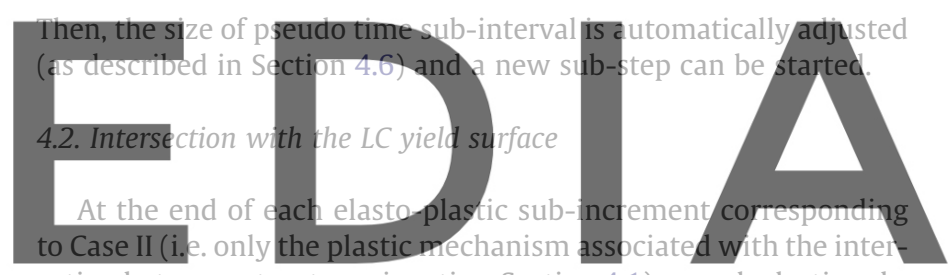

action between structures is active, Section 4.1) or each elastic subincrement (Section 4.5); a check is performed to verify whether the

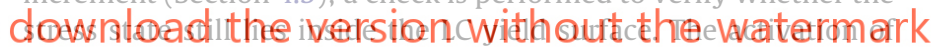

the plastic mechanism associated with macrostructural yielding occurs if

$F_{n-1}=F_{\left|\sigma_{n-1}, r_{n-1}\right|}<0$ and $F_{n}=F_{\left|\sigma_{n}, r_{n}\right|}>0$

In this case, to determine the portion of the stress increment that lies within the yield surface, it is necessary to find a scalar $\mu$ such as [1]:

$\boldsymbol{\sigma}_{\text {in }}=\boldsymbol{\sigma}_{n-1}+\Delta \boldsymbol{\sigma}^{e}(\mu \Delta \boldsymbol{\varepsilon}) \quad(\mathrm{a}) ; \quad F_{\text {in }}=0 \quad$ (b)

The scalar $\mu$ lies within the range $0<\mu<1$. value of $\mu$ equal to zero it implies a purely elasto-plastic deformation during the sub-step, whereas when it is equal to one it indicates a response inside the LC yield surface during the sub-step. In general, $\mu$ gives the portion of the generalised strains sub-increment that moves the stresses from $\boldsymbol{\sigma}_{n-1}$ to the intersection with the yield surface $\boldsymbol{\sigma}_{i n}$. The exact condition (22) (b) is replaced by $\left|F_{i n}\right|<$ FTOL, were FTOL is a small positive tolerance [2]. The value of $\mu$ is obtained solving a non-linear system. The Newton-Rapshon scheme proposed by Sloan [1] has been extended for this model:

$\boldsymbol{\sigma}_{k}=\boldsymbol{\sigma}_{k-1}+\mu_{k} \mathbf{D}_{e} \Delta \boldsymbol{\varepsilon}$
$\mu_{k+1}=\mu_{k}+\Delta \mu_{k+1}$
$\Delta \mu_{k+1}=-\frac{F_{k}}{(\partial F / \partial \boldsymbol{\sigma})_{k}^{T} \Delta \boldsymbol{\sigma}+(\partial F / \partial s)_{k} \Delta s+(\partial F / \partial T)_{k} \Delta T}$

The iterative procedure is started by assuming $\sigma_{0}=\sigma_{n-1}$ and a seed value for $\mu_{1}$, and continues until a relative error in the norm of stresses, defined by the following Equation, is less than some specified tolerance ITOL 
$\frac{\left\|\boldsymbol{\sigma}_{k+1}-\boldsymbol{\sigma}_{k}\right\|}{\left\|\boldsymbol{\sigma}_{k}\right\|}<\mathrm{ITOL}$

Following the procedure proposed in [2], the Newton-Rapshon scheme has been combined with a bisection algorithm used when the Newton-Raphson scheme does not converge after 10 iterations in order to increase the robustness of the procedure. Once (24) is satisfied the stresses and internal variables are updated using (25); and the plastic part of the generalised strains increments $(1-\mu)$ is integrated according to Section 0 , if only the LC plastic mechanism is active, or Section 4.4, if both plastic mechanisms are active

$\boldsymbol{\sigma}_{n}=\boldsymbol{\sigma}_{k} \quad(\mathbf{a}) ; \quad r_{n}=r_{k}$

Sometimes, the LC yield curve can be non-convex, notably in the transition between saturated and unsaturated states [20]. In that case, especial procedures are required, involving, for instance, change of stress variables and smoothing of derivatives. A novel and efficient solution to this problem has been recently proposed [21].

4.3, Integration of the macrostructural elasto-plastic law, BBM (irreversible mechanism associated with the yielding of the macrostructure)

The case of yielding of the macrostructure alone is now considered (Case III). The procedure to integrate the elasto-plastic law is anakgous to that described in Section tic operators (A17), (A18) and (A19) (15) and (17). Once the error criterior gration is fulfilled (i.e. $R_{n} \leqslant$ STOL, wit ses and internal variables are updated -increment stress integration is a whether time is automatically adjusted for the next sub-step. Details of the sub-stepping strategy are presented in Section 4.6.

At the end of each elasto-plastic sub-step it is necessary to

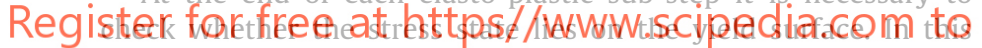
scheme it is possible that the accepted updated stresses (at the end of the sub-increment) do not satisfy the yield criterion, i.e. $|\mathrm{Fn}|>$ FTOL. As such departures are cumulative during the integration process, it is necessary to ensure that the stresses are corrected at the end of each sub-increment in order to satisfy the yield condition and, consequently, update accurately the stresses. This step is sometimes known as 'yield surface drift correction'. The method proposed in [22] has been adopted. The basic assumption is the consideration of a constant total strain during the correction process. In this model, it is also assumed that during the correction process, suction and temperature remain constant. This implies that an elastic strain change must be balanced by an equal and opposite change in the plastic strains.

Following [22,2], the required corrections can be obtained as

$\delta \boldsymbol{\varepsilon}^{e}=\left[\mathbf{D}_{e}\right]^{-1}\left(\boldsymbol{\sigma}_{c}-\boldsymbol{\sigma}_{n}\right) \quad(\mathrm{a}) ; \quad \delta \boldsymbol{\varepsilon}^{p}=\mu_{c} \frac{\partial G}{\partial \boldsymbol{\sigma}}$

Combining (26) (a) and (b) and considering the changes in the hardening parameter, the following set of equations is obtained:

$\boldsymbol{\sigma}_{c}=\boldsymbol{\sigma}_{n}-\mu_{c} \mathbf{D}_{e} \frac{\partial G}{\partial \boldsymbol{\sigma}} \quad(\mathrm{a}) ; \quad r_{c}^{h}=r_{b}^{h}+\mu_{c} \delta r^{h} \frac{\partial G}{\partial \boldsymbol{\sigma}}$

where $\mu_{c}$ is a scalar computed as

$\mu_{c}=\frac{F_{\left|\boldsymbol{\sigma}_{n}, r_{n}^{h}\right|}}{\left(\frac{\partial F}{\partial \boldsymbol{\sigma}}\right)^{T} \mathbf{D}_{e} \frac{\partial G}{\partial \boldsymbol{\sigma}}-\left(\frac{\partial F}{\partial r^{h}}\right)^{T} \delta r^{h} \frac{\partial G}{\partial \boldsymbol{\sigma}}}$

This scheme has to be applied repeatedly until the yield condition $\left(\left|F_{c}\right|<\right.$ FTOL) is satisfied.
4.4. Integration of the elasto-plastic law when two plastic mechanisms are active

When two irreversible mechanisms are active simultaneously, i.e. macrostructural yielding plus one of interaction mechanisms active (Case IV), the integration process used is the same as indicated in Section 4.1, but now the elasto-plastic matrix and vectors correspond to those indicated in the Appendix A for Case IV, i.e. (A20), (A21) and (A22). If the step is accepted, stresses and internal variables are updated. If not, the sub-increment is re-started (see Section 4.6). Finally, at the end of each successful sub-increment, the correction of stresses and internal variables due to the 'yield surface drift' is performed according to a procedure similar to that described previously in Section 4.3.

\subsection{Elastic stress integration}

Due to the formulation of the model, purely elastic stress increments are not very common and they are limited to stress paths that move on the neutral line (Section 2.2) and inside the LC yield surface. For those cases, the stress integration scheme adopted is similar to that described in Section 4.1 but now integration involves the elastic operators (see Appendix A, Eqs. A10, A12 and A13). At the end of the sub-increment, it is checked whether the trial stress state is outside the yield surface (Section 4.2), if not, no plastic yield occurs and the stress state is updated with the trial stress state.

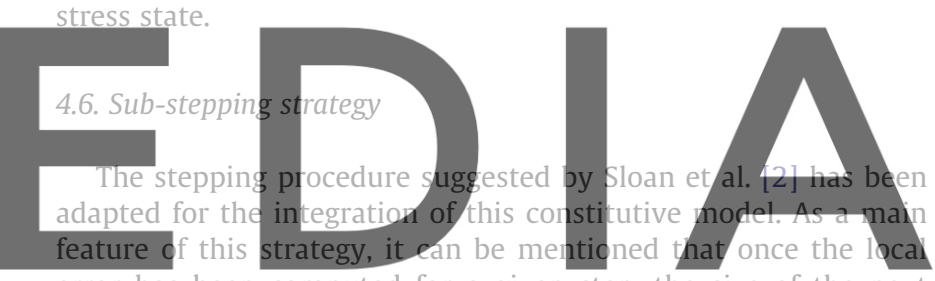
error has been computed for a given step, the size of the next step is evaluated considering the result of this error estimation.

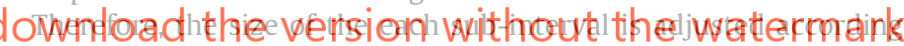
to the information of the previous step. This error control allows the automatic variation of the size of the interval over the course of the integration, depending on the degree of non-1inearity of the model. The sub-increment strategy and the main stages of the integration can be summarized in 10 stages, as follows:

1. The integration scheme is started with the known initial state (stresses and internal variables) and the generalised strains increment.

2. An initial pseudo time step is assumed, $\Delta \tau_{1}$ (a value of 0.5 is typically adopted).

3. The generalized strains sub-increment is obtained from (14).

4. The integration procedure indicated in Section 4.5 is performed in the case of an elastic integration or the one indicated in Sections $4.1,4.3$ or 4.4 in the case of an elastoplastic integration.

5. After a successful elastic sub-increment (Case I), it is necessary to check if the updated stress state remains inside the yield surface (Section 4.2).

- If the sub-increment is purely elastic the stresses and history variables are updated according to Eq. (20). Then the pseudo time is checked according to stage 10.

- If the sub-increment stresses cross the LC yield surface, stresses and internal variables are updated with the elastic portion (25). The plastic part of the sub-increment is integrated according to Sections $4.2,4.3$ or 4.4 depending on which elasto-plastic mechanism(s) is/(are) active. 
6. After a successful elasto-plastic sub-increment and before checking the pseudo time, it is necessary to verify the following points:

- If the sub-increment corresponds to a Case II (i.e. only the interaction mechanism is active), it is necessary to check if the updated stress remains inside the LC yield surface (Section 4.2):

- If the stress remains inside the LC curve, stresses and history variables are updated according to Section 4.1. Then the pseudo time is checked according to stage 10 .

- If the stress sub-increment crosses the LC yield surface, stresses and internal variables are updated with the elastic-plastic portion corresponding to Case II (Section 4.1). The other portion of the elasto-plastic sub-increment, which corresponds to Case IV (i.e. two elato-plastic mechanisms active), is integrated according to Section 4.4

- If the sub-increment corresponds to a Case II or IV, it is necessary to correct for the drift of the yield surface according to Section 4.3. Then the pseudo time is checked according to stage 10 .

The relative error measure $\left(R_{n}\right)$ is computed (19) and $q$ is evaluated as

$q=0.95 \sqrt{\mathrm{STOL} / R_{n}}, \quad$ with the constraint $: 0.2 \leqslant q \leqslant 1.2$

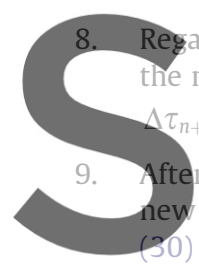

ardless of whether the sub-increment next pseudo time step is auto $q \Delta \tau_{n}$

a refused sub-increment (elastic or elasto-plastic), a sub-increment is re-started with a smaller step-size and with reduced generalizer strains sub-increments according to (14). This loop is repeated until a successful sub-increment size is obtained. Here, an additipnal control

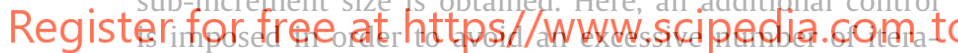

tions. The user can establish a maximum number of iterations to reach a successful integration. On reaching that number, the integration process is aborted and a reduction of the time increment is imposed over the global system. This strategy yields good results, especially when complex highly non-linear problems are modelled.

10. The sub-stepping integration continues until the entire increment of the generalized strains is applied, verified through: $\sum \Delta \tau=\tau=1$

Note that the generalized plasticity model is integrated with the same degree of accuracy and efficiency that the conventional BBM elasto-plastic law. From the algorithm point of view, the main difference is that the 'yield surface drift correction' is omitted in a generalized plasticity model, as no yield surface is explicitly defined.

\section{Application case}

The mechanical model has been widely validated at integration point level in a number of applications [6,7]. In those works, the capabilities of the model to describe and predict the behaviour of expansive soils under complex stress paths have been established. In this paper, the aim is to explore the performance of the model and its implementation in FE when it is applied to the solution of a coupled THM boundary value problem. The case selected involves the THM behaviour of the FEBEX mock-up test, a large-scale heating experiment [23].

The layout of the mock-up test is depicted in Fig. 3. Two electrical heaters (simulating canisters containing heat-emitting waste) were placed in the centre of a steel cylinder $6 \mathrm{~m}$ long and with an inner diameter of $1.62 \mathrm{~m}$. The space between the heaters and the steel cylinder was filled with a 0.64 m-thick engineered barrier made up of blocks of compacted FEBEX bentonite. The barrier is hydrated uniformly from all around the cylinder with an applied

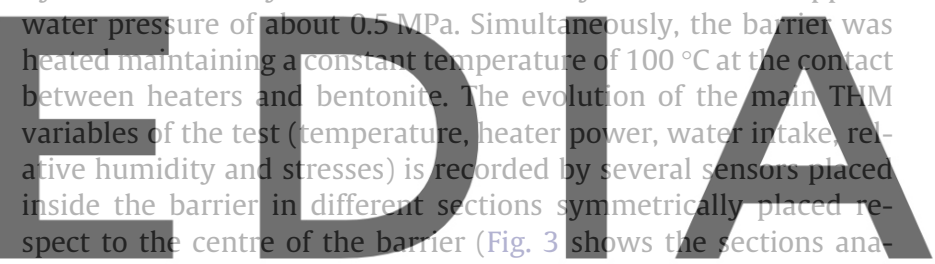

lyzed in this work)

The test has been modelled taking advantage of the basic axial

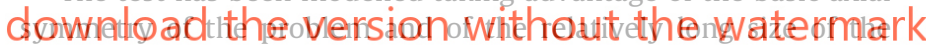
heaters in order to adopt a radial 1-D discretization. All the analyses have been carried out using the computer program CODE_BRIGHT [8]. The geometry of the probiem as weil as the initiai and boundary conditions have been based on [23]. An initial temperature of $20^{\circ} \mathrm{C}$ has been prescribed together with and initial hydrostatic net stress of $0.11 \mathrm{MPa}$ (approximately equal to the weight of the bentonite in the mid diameter of the buffer). Finally, the initial values of relative humidity $(\mathrm{RH})$ recorded in the test have been

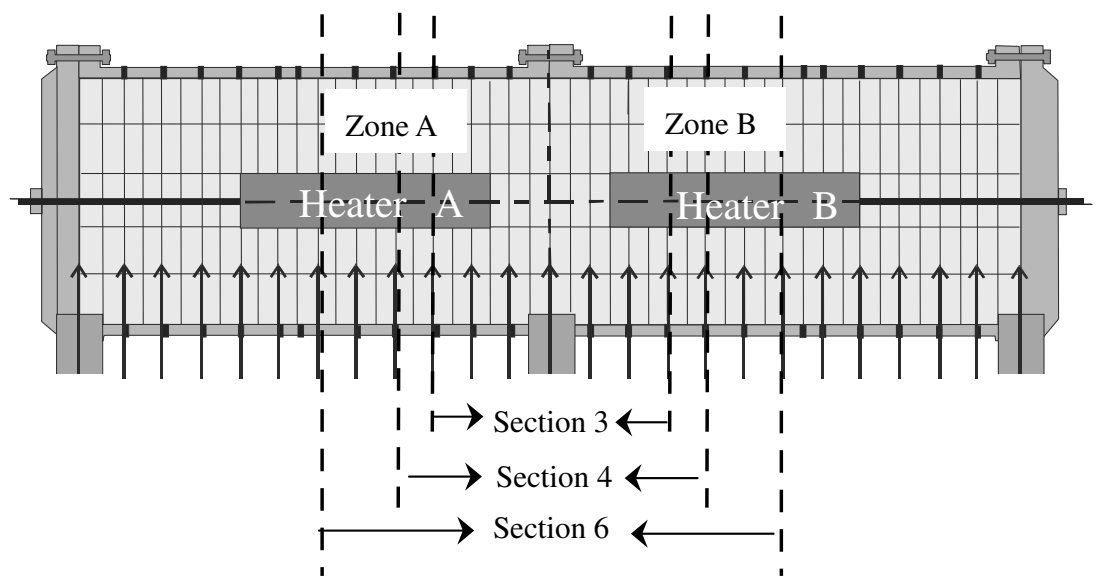

Fig. 3. Layout of the mock-up test. 
used to estimate and prescribe the initial suction. Regarding boundary conditions, a water pressure of $0.55 \mathrm{MPa}$ has been applied on the external part of the bentonite barrier and a stress free condition has been prescribed on the outer boundary of the steel cylinder. Finally, the thermal boundary conditions at a radius equal to $0.15 \mathrm{~m}$ (radial coordinate of the heater elements) are as follows: a staggered increase of heater power has been applied until a temperature of $100{ }^{\circ} \mathrm{C}$ is reached at the contact between heater and bentonite (at 15.6 days), afterwards a constant temperature of $100{ }^{\circ} \mathrm{C}$ has been maintained at this contact point. On the external boundary of the steel cylinder, a thermal radiation condition has been applied [24].

A key feature of this test is that due to simultaneous heating and hydration a series of coupled THM phenomena take place in the clay barrier inducing cycles of wetting and drying in different zones of the buffer. For illustration purposes, Fig. 4 presents the evolution of the RH at two points of a typical section of the barrier. The behaviour of the part of the buffer near heater is very characteristic (sensors at a distance from the axis, $r=0.22 \mathrm{~m}$ ). There is an initial wetting due to the condensation of the vapour coming from the inner barrier regions (values higher than $60 \%$ have been recorded at this stage). However, drying takes place eventually due to the combined effect of temperature increase and reduced vapour arrival. Finally, a gentle hydration is observed due to the inflow of water coming from the outer barrier regions. On the other hand the external part of the barrier (sensors at a distance from the axis, $r=0.70 \mathrm{~m}$ ) is subjected to continuous hydration
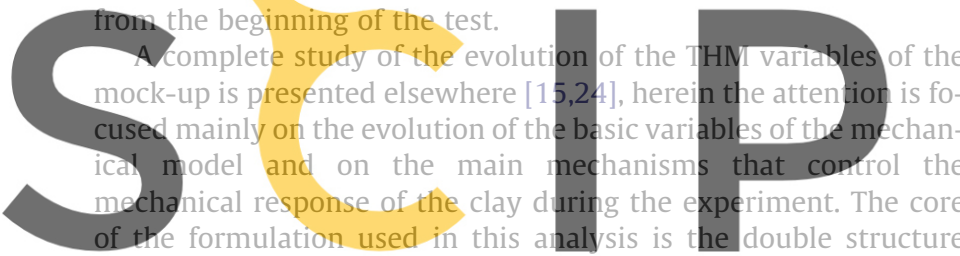

model summarized in the previous sections and presented in detail in [15]. A wide ranging experimental campaign has been carried to

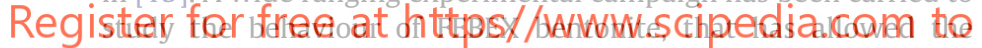
determination of the main parameters of the double structure model [6,24]. Table 2 summarizes the model parameters used in the analysis (more details can be found in [24]). A procedure to determine the main parameters of this model has been presented in [6].

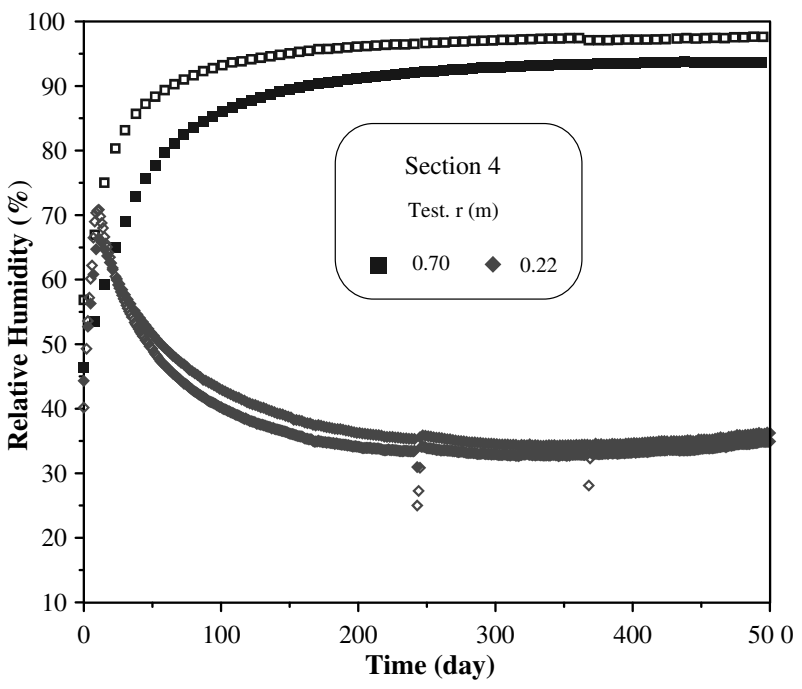

Fig. 4. Evolution of relative humidity in Section 4, mock-up test. Note that for each radial coordinate, the observations of two sensors are plotted, corresponding to the two symmetric halves of the experiment.
Table 2

Parameters used to define the elasto-plastic constitutive law

Parameters defining the Barcelona Basic Model for macrostructural behaviour

$\begin{array}{llllllll}\kappa & \kappa_{\mathrm{s}} & \lambda_{(0)} & r & p_{c}(\mathrm{MPa}) & \zeta\left(\mathrm{MPa}^{-1}\right) & p_{0}^{*}(\mathrm{MPa}) & \alpha_{0}\left({ }^{\circ} \mathrm{C}^{-1}\right)\end{array}$

$\begin{array}{llllllll}0.005 & 0.001 & 0.080 & 0.90 & 0.50 & 0.20 & 5.5 & 1.0 \mathrm{e}^{-5}\end{array}$

Parameters defining the law for microstructural behaviour

$\begin{array}{lll}\alpha_{m}\left(\mathrm{MPa}^{-1}\right) & \beta_{m}\left(\mathrm{MPa}^{-1}\right) & \chi \\ 2.1 \mathrm{e}^{-02} & 2.3 \mathrm{e}^{-03} & 1\end{array}$

Interactions functions

$f_{C}=1+0.9 \tanh \left(20\left(p_{r} / p_{0}\right)-0.25\right) ; \quad f_{S}=0.8-1.1 \tan \mathrm{h}\left(20\left(p_{r} / p_{0}\right)-0.25\right)$

$e_{\text {macro }} \quad e_{\text {micro }}$

0.20

Tolerances

STOL CTOL $\quad$ FTOL

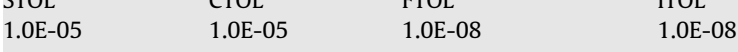

The algorithm described must therefore deal with a wide variety of situations. In order to illustrate the complexity of the couplings between the various THM phenomena and of the interaction between the two structural levels, the behaviour of the two selected points with the same radial coordinates, as in Fig. 4, is now described in some detail using Figs. 5 and 6. Fig. 5a and $b$ present the generalized-stress path on the $p-s$ plane, together with the evolution of $p_{0}$ (apparent preconsolidation stress for the current value of suction) in terms of suction; whereas Fig. 5c and d present the changes of void ratio (macro, micro and

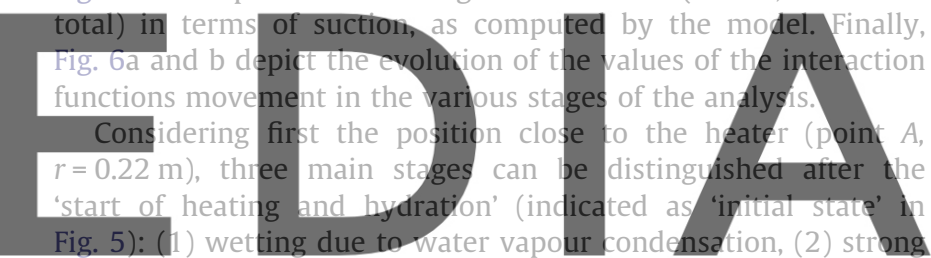

drying induced by the heater, and (3) final hydration. From the beginning the test undergoes wetting (from the external part in-

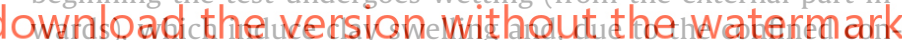
ditions of the test; a global increment of the net stress is computed. Stage '1' (Fig. 5a and c and Fig. 6a is characterized by a suction reduction (due to the condensation of the water vapour) at a very low value of $p$. This implies swelling at both pore levels. The microstructure swells due to a reduction of the generalized microstructural stress (mainly due to suction reduction); and the macrostructure expands by the combined effects of suction reduction and also due to the active interaction mechanism (MS path) that induces plastic macrostructural swelling strains, due to the positive values of the interaction function $f_{s}(4)$. This mechanism implies a softening of the macrostructure with a reduction of $p_{0}^{*}$. Stage ' 2 ' is characterized by strong drying in zones close to the heater. During this stage, hydration progresses in the external zones of the barrier and induces a steady increase of the stress level. Those two facts cause a rise of the generalized microstructural stress and, consequently, a contraction of the microstructure (MC path). The coupling mechanism between micro and macro structure induces positive (contractive) plastic macrostructural strains due to the positive values of the interaction function $f_{c}$. The macrostructure also contracts due to the combined effect of $p$ and suction increments. So, this stage implies a macrostructural hardening. Finally, stage ' 3 ' is related to the final hydration stage of this zone, controlled by a gradual reduction of suctions and a significant increase of $p$. During the MS path the interaction function $f_{s}$ has negative values, which implies a hardening of the macrostructure associated to a wetting process under high pressure [4]. At a suction close to $110 \mathrm{MPa}$, the stress path reaches the LC curve and drags it along until the end of the analysis. 

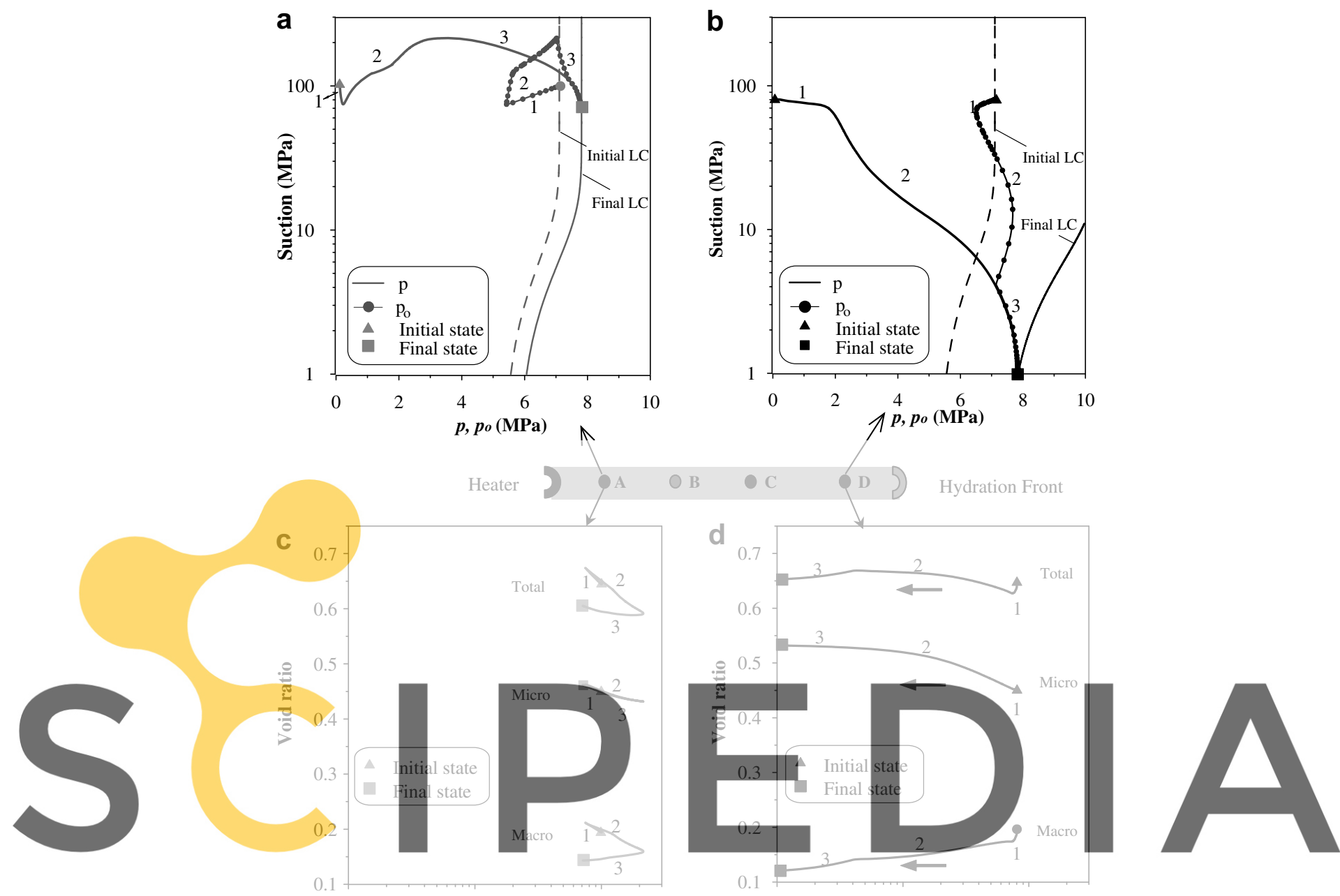

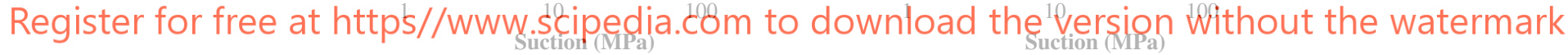

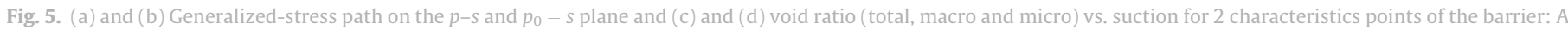
$(r=0.22 \mathrm{~m})$ and $\mathrm{D}(r=0.70 \mathrm{~m})$.
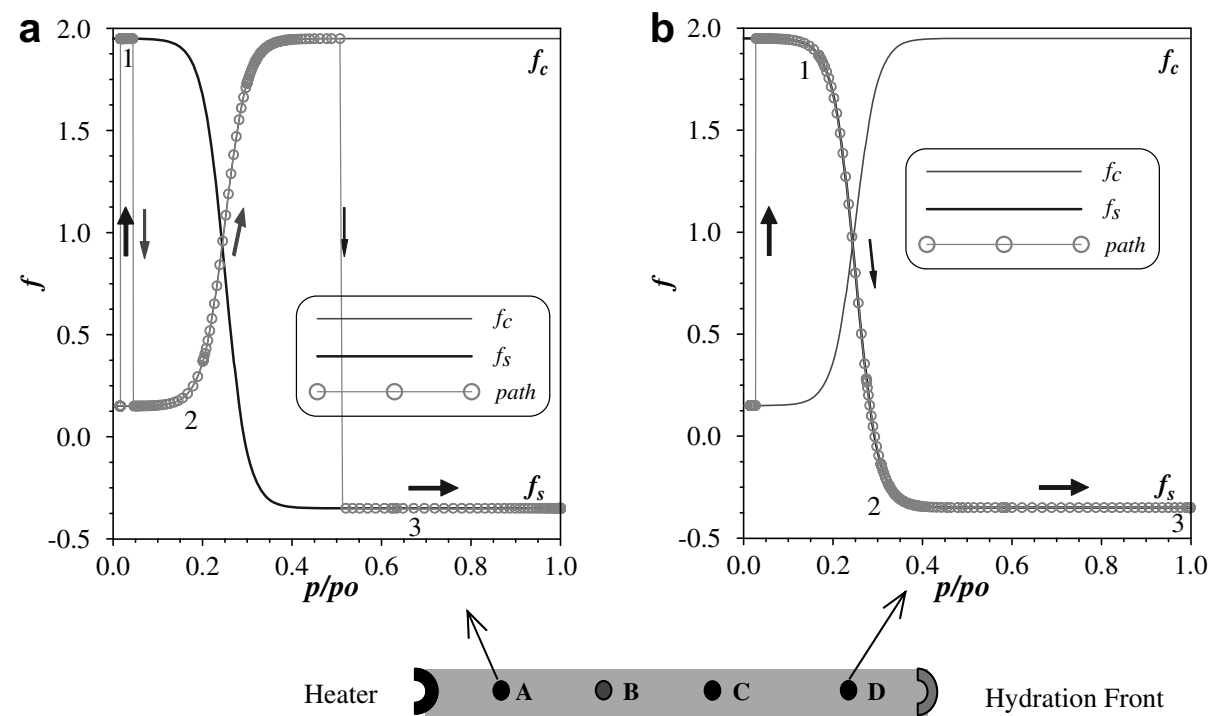

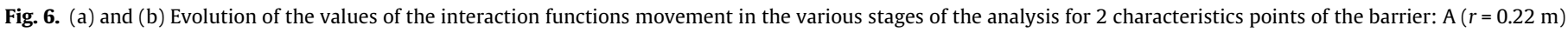
and $\mathrm{D}(r=0.70 \mathrm{~m})$. 
Close to the hydration boundary (point $D, r=0.70 \mathrm{~m}$ ), a quite different behaviour is observed (Fig. 5b and $d$ and Fig. 6b). Three main stages have been identified here as well. Stage ' 1 ' is characterized by a slight suction reduction (the hydration front has not reach this zone yet) and a noticeable stress increment given by the progressive hydration of the external part of the barrier. During this stage the main compression corresponds to the closing of the macropores due to the dominant effect of the stress increment. The microstructure expands, due to reduction of the microstructural effective stress (3a). Due to the positive values of the interaction function fs during the MS path a macrostructural softening (reduction of $\left.p_{0}^{*}\right)$ is predicted. Stage ' 2 ' maintains this tendency to suction reduction and stress increase, but now the suction reduction is more noticeable (the hydration front is reaching this zone). This implies a large microstructural swelling due to the strong reduction of microstructural effective stress (3a). This microstructural expansion, under confined conditions, tends to increase the stress level and compresses even more the macropores. Finally, stage'3' starts when the stress path reaches the LC curve. After that, the stress path drags the LC curve with it and both move together. The shape of the stress path in this last stage is mainly controlled by the LC curve. During this stage the macropores reduction takes place under small changes of stresses, i.e. a kind of macrostructural collapse occurs in this zone of the barrier at advanced phases of hydration.

Comparing Fig 5a and b it can be observed that the generalized stress paths are quite different. In addition, there is an important increment of the elastic domain in zones corresponding to point 'D' ('Final LC' in Fig. 5b). The hardening of the materiai is due to the reduction of macropores induded, tural expansion during hydration coupling between micro and macr s anism to model this behaviour. T to a macropore reduction under confin
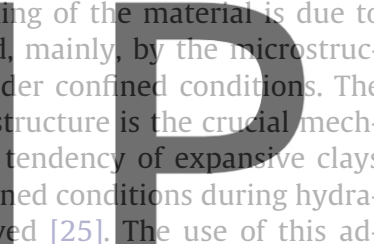
vanced model that allows the inclusion of the soil fabric effects in the analysis it is a crucial aspect to describe the complex clay

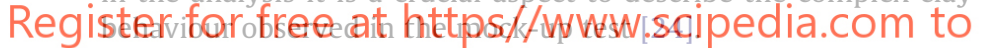

The performance of the model was very satisfactory. For illustration, two additional figures showing comparisons between experiment and model results are presented below. Fig. 7a shows the evolution of radial stress for two typical sections (Sections 3A\& B and 6A\& B, see Fig. 3). As it can be observed, the model reproduces quite well the quick increase of radial stress at the beginning of the hydration and also the maximum value of radial stress observed in the test. Fig. 7b also shows that the model is able to capture reasonably well the evolution of the RH during the first complex stages of the test. More details of the mock-up test modelling can be found in [24]. Complex THM behaviour has thus been successfully tracked using a generalized plasticity constitutive law for a double structure material and the implementation algorithm described in the paper.

To check the accuracy and efficiency of the proposed algorithm, the same analysis has been performed using decreasing values of the stress tolerances STOL and CTOL that are assumed equal. The rest of model parameters and tolerances have maintained unchanged (Table 2). Table 3 presents the following variables for the different computations carried out: 'CPU time', 'Radial stress, 'Total sub-steps' and 'Stress error'. The numerical analyses have been performed using A Sillicon Graphics Origin 300 (8 processors MIPS R14000 at $600 \mathrm{MHz}, 6$ GB RAM memory) computer. The CPU time recorded is not for the integration algorithm alone but for the entire computation. The 'Stress error' ( $\left.\sigma_{\text {error }}\right)$ has been evaluated according to $[2]$

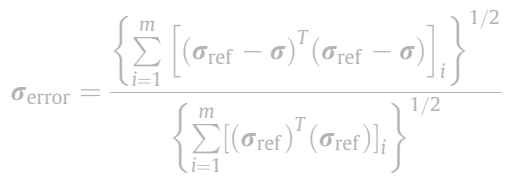

where the subscript $i$ refers to each integration point, $m$ is the total number of integration points in the mesh, $\sigma$ are the stresses com-

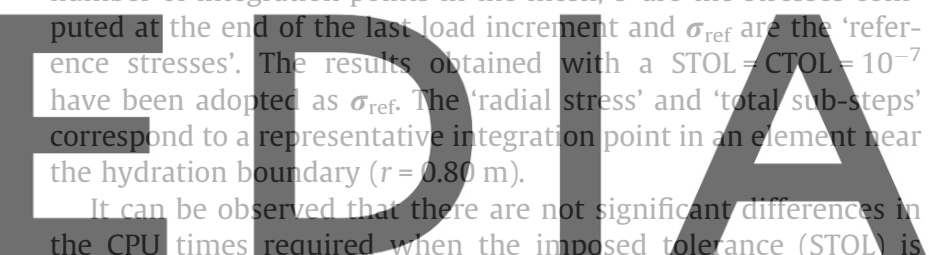

higher than $10^{-4}$; while the CPU times required for tolerances below this value increase dramatically. This implies that when low

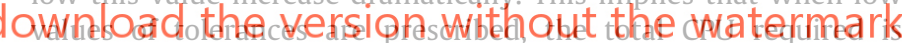
mainly spent in the stress integration process, as it can be observed in the number of total steps required. As the tolerances are reduced an asymptotic tendency for a slight radial stress reduction is computed. As it should be expected, the accuracy of the algorithm increases as the tolerances are reduced but quite small errors are still obtained with the larger stress tolerances, with the resulting savings in CPU times.
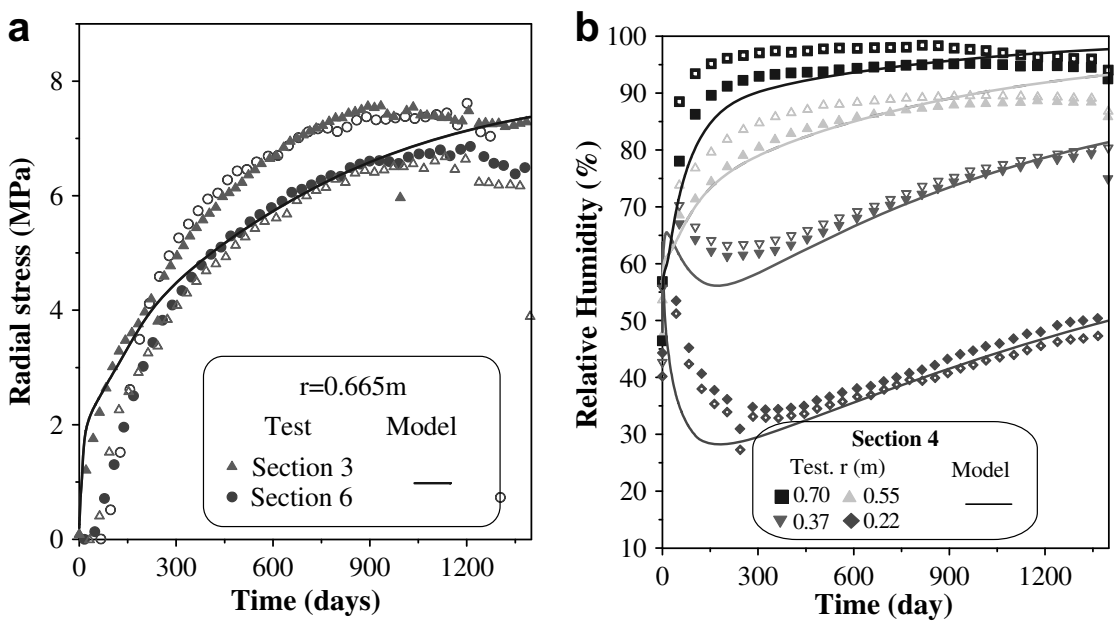

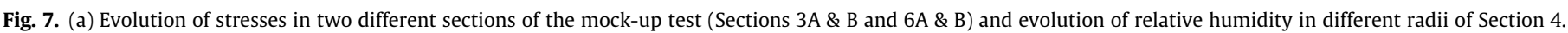


Table 3

Effect of stress tolerances on algorithm performance and stress error

\begin{tabular}{lllll}
\hline STOL $=$ CTOL & $\begin{array}{l}\text { CPU time } \\
(\mathrm{s})\end{array}$ & $\begin{array}{l}\text { Radial stress } \\
(\mathrm{MPa})\end{array}$ & $\begin{array}{l}\text { Total sub } \\
\text { steps }\end{array}$ & $\begin{array}{l}\text { Stress error } \\
\boldsymbol{\sigma}_{\text {error }}\end{array}$ \\
\hline $1.0 \mathrm{E}-01$ & 1614 & 7.91221 & 13416 & $83.9 \mathrm{E}-04$ \\
$1.0 \mathrm{E}-02$ & 1614 & 7.91221 & 13416 & $83.9 \mathrm{E}-04$ \\
$1.0 \mathrm{E}-03$ & 1616 & 7.91220 & 13450 & $83.2 \mathrm{E}-04$ \\
$1.0 \mathrm{E}-04$ & 1620 & 7.91216 & 13452 & $81.5 \mathrm{E}-04$ \\
$1.0 \mathrm{E}-05$ & 1787 & 7.90938 & 15999 & $4.87 \mathrm{E}-04$ \\
$1.0 \mathrm{E}-06$ & 2437 & 7.90680 & 25503 & $1.57 \mathrm{E}-04$ \\
$1.0 \mathrm{E}-07$ & 4518 & 7.90549 & 52100 & $0.00 \mathrm{E}+00$ \\
\hline
\end{tabular}

\section{Conclusions and final remarks}

In this paper an algorithm to implement a double structure model for expansive clays in a finite element code has been presented. The algorithm is based on Sloan's scheme [1,2] and has been specifically adapted to the characteristics of this model. The algorithm has been extended to update stresses and internal variables in terms of the increment in generalized strains that include strains, suction and temperature. Another extension of the scheme is related to the handling of more than one irreversible mechanism. The same modified scheme has been used to integrate the two elasto-plastic laws (one based on classical plasticity and one based on generalized plasticity) and the highly non-linear elastic law. This ensures the integration of the different parts of the model with the same degree of accuracy and efficiency. The performance of the model in the application case presented herein has been very satisfactory with a good level of efficiency and robustness. A good reproduction of a complex THM case has been achieved and, in addition, a physical interpretation of the observed behaviour within the context of double structure media has also been obtained. Most of the main features of the behaviour are correctly reproduced by the model allowing a more detailed examination of the role that the different variables play in relation to the overall behaviour of the clay barrier submitted to heating and hydration under confined conditions. A sensitivity analysis has illustrated the dependency of the efficiency and accuracy of the algorithm with the values of stress tolerances adopted.

\section{Acknowledgements}

The authors would like to acknowledge ENRESA for funding this work. The projects FEBEX and NF-PRO are partially funded by the European Commission. The authors are also grateful for the financial support given by $\mathrm{CNPq}$ (Conselho Nacional de Desenvolvimento Cientifico e Tecnologico, Brazil) and by the Ministerio de Ciencia y Tecnología of Spain through research Grant BIA2005-05801 and BIA2008-06537.

\section{Appendix A. Mechanical model}

The BBM yield surface $\left(F_{\mathrm{LC}}\right)$ is given by $(3)$ and the plastic potential $(G)$ is expressed as

$G=\alpha 3 J^{2}-\left[\frac{g(\theta)}{g\left(-30^{\circ}\right)}\right]^{2} M^{2}\left(p+p_{s}\right)\left(p_{0}-p\right)=0$

where $\alpha$ is determined according to [12]. The dependence of the tensile strength on suction and temperature is given by

$p_{s}=k s e^{-\rho \Delta T}$

where $k$ and $\rho$ are model parameters. The dependence of $p_{0}$ on suction and temperature is given by $p_{0}=p_{c}\left(\frac{p_{0 T}^{*}}{p_{c}}\right)^{\frac{\lambda_{(0)}-\kappa}{\lambda_{(s)}}} \quad(\mathrm{a}) ;$

$p_{0 T}^{*}=p_{0}^{*}+2\left(\alpha_{1} \Delta T+\alpha_{3} \Delta T|\Delta T|\right)$

where $p_{c}$ is a reference stress, $\alpha_{1}$ and $\alpha_{3}$ are models parameters. $\lambda_{(s)}$ is the compressibility parameter for changes in net mean stress for virgin states of the soil. This parameter depends on suction according to

$\lambda_{(s)}=\lambda_{(0)}[r+(1-r) \exp (-\zeta s)]$

where $r$ is a parameter which defines the minimum soil compressibility (at infinite suction) and $\zeta$ is a parameter which controls the rate of decrease of soil compressibility with suction. The macrostructural bulk modulus $\left(K_{M}\right)$ for changes in mean stress is evaluated with the following law:

$K_{M}=\frac{\left(1+e_{M}\right)}{\kappa} p$

The microstructural bulk modulus $\left(K_{1}\right)$ is evaluated as follows:

$K_{m}=\frac{e^{-\alpha_{m} \hat{p}}}{\beta_{m}}$

where $\alpha_{m}$ and $\beta_{m}$ are model parameters. The shear modulus $G_{t}$ is obtained from a linear elastic model as follows:

$G_{t}=\frac{3(1-2 \mu) K}{2(1+\mu)}$

where $\mu$ is the Poisson's coefficient. The macrostructural bulk modulus for changes in suction is computed considering the following law:

$K_{s}=\frac{\left(1+e_{M}\right)\left(s+p_{\mathrm{atm}}\right)}{\kappa_{s}}$

where $\kappa_{s}$ is the macrostructural elastic stiffness parameter for changes in suction. The macrostructural bulk modulus for changes in suction is computed considering the following law:

$K_{T}=\frac{1}{\left(\alpha_{0}+\alpha_{2} \Delta T\right)}$

where $\alpha_{0}$ and $\alpha_{2}$ are parameters related to the elastic thermal strain.

Elastic and elasto-plastic relationships.

Firstly, the elastic tensors related to Eq. (8) are presented. These tensors correspond to the Case identified as ' $\mathrm{I}$ ' (Elastic sub-increment) in Section 4. The elastic matrix is evaluated as follows:

$\mathbf{D}_{e}=\left[\begin{array}{cccccc}K+\frac{4}{3} G_{t} & K-\frac{2}{3} G_{t} & K-\frac{2}{3} G_{t} & 0 & 0 & 0 \\ & K+\frac{4}{3} G_{t} & K-\frac{2}{3} G_{t} & 0 & 0 & 0 \\ & & K+\frac{4}{3} G_{t} & 0 & 0 & 0 \\ & & & G_{t} & 0 & 0 \\ \text { symm } & & & & G_{t} & 0 \\ & & & & & G_{t}\end{array}\right]$

where $K$ is the global bulk that may be computed considering the elastic contribution of the two pores structures, the global stiffness is defined as

$K=\left(\frac{1}{K_{M}}+\frac{1}{K_{m}}\right)^{-1}$

$\alpha_{s}=\left(\frac{1}{K_{s}}+\frac{\chi}{K_{m}}\right) \mathbf{D}_{e} \cdot \mathbf{m}$

$\alpha_{T}=\frac{1}{K_{T}} \mathbf{D}_{e} \cdot \mathbf{m}$

More details can be found in [7]. The elasto-plastic tensors related to (9) correspond to three different possibilities (Case II, III and IV) indicated in Section 4, which introduced as follows: 
- Case II: when only the plastic mechanism associated with the coupling between structures is active:

$$
\begin{aligned}
& \mathbf{D}_{e p}=\mathbf{D}_{e} \cdot\left[\mathbf{I}-\frac{1}{\bar{H}_{\beta}} \mathbf{m}_{\beta} \cdot \mathbf{n}_{\beta}^{T} \cdot \mathbf{D}_{e}\right] \\
& \gamma_{s}=\left(\alpha_{s}+\beta_{s}\right)-\frac{1}{\bar{H}_{\beta}} \mathbf{D}_{e} \cdot \mathbf{m}_{\beta} \mathbf{n}_{\beta}^{T} \cdot\left(\alpha_{s}+\beta_{s}\right) \\
& \gamma_{T}=\alpha_{T}-\frac{1}{\bar{H}_{\beta}} \mathbf{D}_{e} \cdot \mathbf{m}_{\beta} \mathbf{n}_{\beta}^{T} \cdot \alpha_{T}
\end{aligned}
$$

- Case III: when only the plastic mechanism associated with the yielding of the macrostructure is active:

$$
\begin{aligned}
& \mathbf{D}_{e p}=\mathbf{D}_{e} \cdot\left[\mathbf{I}-\frac{1}{\bar{H}_{L C}} \mathbf{m}_{L C} \cdot \mathbf{n}_{L C}^{T} \cdot \mathbf{D}_{e}\right] \\
& \gamma_{s}=\alpha_{s}-\frac{1}{\bar{H}_{L C}} \mathbf{D}_{e} \cdot \mathbf{m}_{L C}\left(l+\mathbf{n}_{L C}^{T} \cdot \alpha_{s}\right) \\
& \gamma_{T}=\alpha_{T}-\frac{1}{\bar{H}_{L C}} \mathbf{D}_{e} \cdot \mathbf{m}_{L C}\left(d+\mathbf{n}_{L C}^{T} \cdot \alpha_{T}\right)
\end{aligned}
$$

- Case IV: when two plastic mechanisms are active:

$\mathbf{D}_{e p}$

$$
=\mathbf{D}_{e} \cdot\left[\mathbf{I}-\frac{1}{|\overline{\mathbf{H}}|}\left(\bar{H}_{\beta} \mathbf{m}_{L C} \cdot \mathbf{n}_{L C}^{T}-\bar{h}_{\beta} \mathbf{m}_{L C} \cdot \mathbf{n}_{\beta}^{T}+\bar{H}_{L C} \mathbf{m}_{\beta} \cdot \mathbf{n}_{\beta}^{T}-\bar{h}_{L C} \mathbf{m}_{\beta} \cdot \mathbf{n}_{L C}^{T}\right) \cdot \mathbf{D}_{e}\right]
$$

$$
\begin{aligned}
\gamma_{s}= & \alpha_{s}+\beta_{s}-\frac{1}{|\overline{\mathbf{H}}|} \mathbf{D}_{e} \cdot\left\{\bar{H}_{\beta} \mathbf{m}_{L C}\left[l+l_{\beta}+\mathbf{n}_{L C}^{T} \cdot\left(\alpha_{s}+\beta_{s}\right)\right]\right. \\
& -\bar{h}_{\beta} \mathbf{m}_{L C} \mathbf{n}_{\beta}^{T} \cdot\left(\alpha_{s}+\beta_{s}\right)+\bar{H}_{L C} \mathbf{m}_{\beta} \mathbf{n}_{\beta}^{T} \cdot\left(\alpha_{s}+\beta_{s}\right) \\
& \left.-\bar{h}_{L C} \mathbf{m}_{\beta}\left[l+l_{\beta}+\mathbf{n}_{L C}^{T} \cdot\left(\alpha_{s}+\beta_{s}\right)\right]\right\}
\end{aligned}
$$

$$
\begin{aligned}
\gamma_{T}= & \alpha_{T}-\frac{1}{|\overline{\mathbf{H}}|} \mathbf{D}_{e} \\
& \cdot\left[\bar{H}_{\beta} \mathbf{m}_{L C}\left(d+\mathbf{n}_{L C}^{T} \cdot \alpha_{T}\right)-\bar{h}_{\beta} \mathbf{m}_{L C} \mathbf{n}_{\beta}^{T} \cdot \alpha_{T}+\bar{H}_{L C} \mathbf{m}_{\beta} \mathbf{n}_{\beta}^{T} \cdot \alpha_{T}\right. \\
& \left.-\bar{h}_{L C} \mathbf{m}_{\beta}\left(d+\mathbf{n}_{L C}^{T} \cdot \alpha_{T}\right)\right]
\end{aligned}
$$

where the definition of these variables are as follows:

$$
\begin{aligned}
& l_{L C}=\frac{\partial F_{L C}}{\partial s} \\
& d=\frac{\partial F_{L C}}{\partial T} \\
& \bar{H}=\frac{\partial F_{L C}}{\partial p_{0}} \frac{\partial p_{0}}{\partial p_{0}^{*}} \frac{\partial p_{0}^{*}}{\partial \boldsymbol{\varepsilon}_{v}^{p}} \\
& \dot{\boldsymbol{\varepsilon}}_{v L C}^{p}=\dot{\lambda}_{L C} \mathbf{m}^{T} \cdot \mathbf{m}_{L C} \\
& \dot{\boldsymbol{\varepsilon}}_{v \beta}^{p}=\dot{\lambda}_{\beta} \mathbf{m}^{T} \cdot \mathbf{m}_{\beta} \\
& \bar{H}_{L C}=H_{L C}+H_{L C}^{c}=H_{L C}+\mathbf{n}_{L C}^{T} \cdot \mathbf{D}_{e} \cdot \mathbf{m}_{L C} \\
& \bar{h}_{\beta}=h_{\beta}+h_{\beta}^{c}=h_{\beta}+\mathbf{n}_{L C}^{T} \cdot \mathbf{D}_{e} \cdot \mathbf{m}_{\beta} \\
& \dot{e}_{L C}=\mathbf{n}_{L C}^{T} \cdot \mathbf{D}_{e} \cdot \dot{\boldsymbol{\varepsilon}} \\
& \dot{s}_{L C}=\left[l_{L C}+l_{\beta}+\mathbf{n}_{L C}^{T} \cdot\left(\alpha_{s}+\beta_{S}\right)\right] \dot{s} \\
& \dot{t}_{L C}=\left(d+\mathbf{n}_{L C}^{T} \cdot \alpha_{T}\right) \dot{T} \\
& \beta_{S}=-\frac{1}{H_{\beta}} \omega_{\beta} \chi \mathbf{D}_{e} \cdot \mathbf{m}_{\beta} \\
& H_{L C}=-\bar{H}_{\mathbf{m}^{T}} \cdot \mathbf{m}_{L C} \\
& h_{\beta}=-\bar{H} \mathbf{m}^{T} \cdot \mathbf{m}_{\beta} \\
& l_{\beta}=-\frac{1}{H_{\beta}} h_{\beta} \omega_{\beta} \chi
\end{aligned}
$$

$\bar{h}_{L C}=h_{L C}^{c}=\mathbf{n}_{\beta}^{T} \cdot \mathbf{D}_{e} \cdot \mathbf{m}_{L C}$

$\bar{H}_{\beta}=H_{\beta}+H_{\beta}^{c}=H_{\beta}+\mathbf{n}_{\beta}^{T} \cdot \mathbf{D}_{e} \cdot \mathbf{m}_{\beta}$

$\dot{e}_{\beta}=\mathbf{n}_{\beta}^{T} \cdot \mathbf{D}_{e} \cdot \dot{\boldsymbol{\varepsilon}}$

$\dot{s}_{\beta}=\mathbf{n}_{\beta}^{T} \cdot\left(\alpha_{s}+\beta_{s}\right) \dot{s}$

$\dot{t}_{\beta}=\mathbf{n}_{\beta}^{T} \cdot \alpha_{T} \dot{T}$

Stress invariants

$p=\left(\frac{1}{3}\right)\left(\sigma_{x}+\sigma_{y}+\sigma_{z}\right)$

$J^{2}=1 / 2$ trace $\left(s^{2}\right)$

$\theta=-\frac{1}{3} \sin ^{-1}\left(1.5 \sqrt{3} \operatorname{det} s / J^{3}\right)$

$s=\boldsymbol{\sigma}-p \mathbf{I}$

\section{References}

[1] Sloan S. Sub-stepping schemes for the numerical integration of elastoplastic stress-strain relations. Int J Numer Meth Eng 1987;24: 893-911.

[2] Sloan S, Abbo A, Sheng D. Refined explicit integration of elastoplastic models with automatic error control. Eng Comput 2001;18(1/2):121-54.

[3] Gens A, Alonso EE. A framework for the behaviour of unsaturated expansive clays. Canad Geotech J 1992;29:1013-32.

[4] Alonso E, Vaunat J, Gens A. Modelling the mechanical behaviour of expansive clays. Eng Geology 1999;54:173-83.

[5] Alonso E. Modelling expansive soil behaviour. In: Proceeding of 2nd international conference on unsaturated soils, vol. 1, Beijing, China; 1998. p. 37-70.

[6] Lloret A, Villar MV, Sánchez M, Gens A, Pintado X, Alonso EE. Mechanical behaviour of heavily compacted bentonite under high suction changes. Géotechnique 2003;53(1):27-40.

[7] Sánchez M, Gens A, Guimarães L, Olivella S. A double structure generalized plasticity model for expansive materials. Int J Numer Anal Meth Geomech 2005;29:751-87.

[8] Olivella S, Gens A, Carrera J, Alonso EE. Numerical formulation for a simulator (CODE-BRIGHT) for the coupled analysis of saline media. Eng Comput 1996;13(7):87-112.

[9] Pastor M, Zienkiewicz O, Chan A. Generalized plasticity and the modelling of soil behaviour. Int J Numer Anal Meth Geomech 1990;14:151-90.

[10] Argyris JH, Faust G, Szimmat J, Warnke EP, Willam KJ. Recent developments in the finite element analysis of prestressed concrete reactor vessels. Nucl Eng Design 1974;28:42-75.

[11] Sheng D, Sloan SW, Yu HS. Aspects of finite element implementation of critical state models. Comput Mech 2000;26:185-96.

[12] Alonso E, Gens A, Josa A. A constitutive model for partially saturated soils. Géotechnique 1990;40(3):405-30.

[13] Eekelen Van. HAM Isotropic yield surfaces in three dimensions for use in soil mechanics. Int J Num Anal Meth Geomech 1980;4:89-101.

[14] Gens A. Constitutive laws. Modern issues in non-saturated soils. Springer; 1995. p. 129-58.

[15] Sánchez M. Thermo-Hydro-Mechanical coupled analyses in low permeability media. PhD thesis, Universitat Politècnica de Catalunya, Spain; 2004.

[16] Rizzi E, Giulio M, Willam K. On failure indicators in multi-dissipative materials. Int J Sol Struct 1996;33(20-22):3124-87.

[17] Erratum: Eng Comput, 19(5/6); 2002. p. 594-594.

[18] Potts D, Zdravković L. Finite element analysis in geotechnical engineering. Theory. Bath: Thomas Telford; 1999.

[19] Sheng D, Sloan S, Gens A, Swith W. Finite element formulation and algorithms for unsaturated soils. Part I: Theory. Int J Numer Anal Meth Geomech 2003;27:745-65

[20] Sheng D. Non convexity of the Barcelona Basic Model - comment on S.J Wheeler, D. Gallipoli and M. Karstunen (2002:26:1561-1571). Int J Num Anal Methods in Geomech 2003;27:879-81.

[21] Sheng DC, Pedroso D, Abbo AJ. In: Proceeding of 1st European conference on unsaturated soils, Durham, UK; 2008. p. 705-11.

[22] Potts D, Gens A. A critical assessment of methods of correcting for drift from the yield surface in elastoplastic finite element analysis. Int J Num Anal Meth Geomech 1985;9:149-59.

[23] Martin PL, Barcala JM. Large scale buffer material test: mock-up experiment at CIEMAT. Eng Geology 2005;81(3):298-316.

[24] Sánchez M, Gens A. FEBEX II final report on THM modelling. Barcelona: ENRESA-70-UPC-L-5-015; 2005.

[25] Lloret A, Villar MV, Romero E. Final Report on THM laboratory tests. Madrid: ENRESA-CIEMAT/DIAE/54520/3/04; 2004. 